In vivo molecular imaging of colorectal cancer using quantum dots targeted to vascular endothelial growth factor receptor 2 and optical coherence tomography/laser-induced fluorescence dual-modality imaging

Jordan L. Carbary-Ganz

Weston A. Welge

Jennifer K. Barton

Urs Utzinger 


\title{
In vivo molecular imaging of colorectal cancer using quantum dots targeted to vascular endothelial growth factor receptor 2 and optical coherence tomography/ laser-induced fluorescence dual-modality imaging
}

\author{
Jordan L. Carbary-Ganz, Weston A. Welge, Jennifer K. Barton, and Urs Utzinger* \\ University of Arizona, Biomedical Engineering, Thomas W. Keating Bioresearch Building, 1657 E Helen Street, Tucson, \\ Arizona 84721, United States
}

\begin{abstract}
Optical coherence tomography/laser induced fluorescence (OCT/LIF) dual-modality imaging allows for minimally invasive, nondestructive endoscopic visualization of colorectal cancer in mice. This technology enables simultaneous longitudinal tracking of morphological (OCT) and biochemical (fluorescence) changes as colorectal cancer develops, compared to current methods of colorectal cancer screening in humans that rely on morphological changes alone. We have shown that QDot655 targeted to vascular endothelial growth factor receptor 2 (QD655-VEGFR2) can be applied to the colon of carcinogen-treated mice and provides significantly increased contrast between the diseased and undiseased tissue with high sensitivity and specificity ex vivo. QD655-VEGFR2 was used in a longitudinal in vivo study to investigate the ability to correlate fluorescence signal to tumor development. QD655-VEGFR2 was applied to the colon of azoxymethane (AOM-) or salinetreated control mice in vivo via lavage. OCT/LIF images of the distal colon were taken at five consecutive time points every three weeks after the final AOM injection. Difficulties in fully flushing unbound contrast agent from the colon led to variable background signal; however, a spatial correlation was found between tumors identified in OCT images, and high fluorescence intensity of the QD655 signal, demonstrating the ability to detect VEGFR2 expressing tumors in vivo. @ 2015 Society of Photo-Optical Instrumentation Engineers (SPIE) [DOI: 10.1117/1.JBO.20.9.096015]
\end{abstract}

Keywords: quantum dots; molecular imaging; colorectal cancer; in vivo; optical coherence tomography; fluorescence spectroscopy; dual-modality; microendoscope.

Paper 150300R received May 21, 2015; accepted for publication Aug. 17, 2015; published online Sep. $23,2015$.

\section{Introduction}

Advances in in vivo imaging of the molecular expression of cancer have provided access to understanding the tumor microenvironment and how variations in expression of proteins and signaling molecules affect cancer development and treatment. ${ }^{1}$ The ability to visualize the molecular information of tumors has the potential to aid in surgical removal of lesions, ${ }^{2,3}$ provide a route for personalized treatment and treatment monitoring, 4,5 and provide a method of early detection of a lesion. ${ }^{6,7}$ Colorectal cancer is the third most commonly diagnosed and third most deadly cancer in the United States, accounting for $8 \%$ of cases in both categories, with mouse models being critical tools in its study. ${ }^{8}$ Currently, many studies use xenograft models of cancer, allowing for the selection of cell types (and thus molecular expression) present and for the placement of the tumor in an area accessible to imaging. ${ }^{9-13}$ Xenograft models, however, have many deficiencies, including limited relevance to spontaneous carcinogenesis, or to the natural tumor microenvironment. ${ }^{14,15}$ The carcinogen azoxymethane (AOM) has been shown to cause the sporadic growth of colorectal tumors in the distal colon of mice, exhibiting many of the morphological and pathological features associated with sporadic

*Address all correspondence to: Urs Utzinger, E-mail: utzinger@email.arizona .edu colorectal cancer in humans. ${ }^{16}$ This model can be used to study the tumors of the colon in vivo, providing a physiologically relevant method for studying colorectal cancer and its detection.

Targeted contrast agents have great potential to detect cancer specific biochemicals in vivo, allowing for earlier detection of the disease rather than morphological changes alone. Quantum dots are nanoscopic particles of semiconductors with fluorescence emission affected by the size of the particle. They have many desirable fluorescence qualities for biological imaging, such as high quantum efficiency, high photostability, a wide range of excitation wavelengths, a narrow emission band, and they can be produced to emit over a wide range of wavelengths. ${ }^{17-21}$ Many studies have used quantum dots as fluorescent markers for cancer in vitro ${ }^{22-27}$ and in animal models in vivo, ${ }^{28-40}$ with their use in vivo being mostly limited to nontargeted $^{29,34,37}$ or xenograft ${ }^{28,30-33,38-40}$ labeling. While numerous molecular targets of carcinogenesis exist, it has been shown that vascular endothelial growth factor receptor 2 (VEGFR2) is upregulated in many cancers, including some colorectal cancers, as it is important in tumor angiogenesis. ${ }^{41-45}$ Also, VEGFR2 is currently considered a predictor for clinical outcome and, in some instances, is used in targeted therapy with antiangiogenic drugs. ${ }^{46-48}$ Recently, our group demonstrated

$1083-3668 / 2015 / \$ 25.00$ @ 2015 SPIE 
that QDot655 targeted to VEGFR2 (QD655-VEGFR2) can be applied to the colon of carcinogen-treated mice in vivo and provides significantly increased contrast between diseased and undiseased tissue ex vivo (explanted) with high sensitivity and specificity compared to a control contrast agent. ${ }^{49}$ This quantum dot is readily available, easy to conjugate, emits in a region of light that has less endogenous emission interference, and is compatible with all commonly used optical coherence tomography (OCT) systems. Thus, evidence exists that quantum dots bioconjugated to VEGFR2 antibodies can provide a mechanism for evaluating the molecular changes of colorectal tumors in vivo.

Optical imaging of ex vivo and xenograft tissue, as well as in vitro models, allows for high resolution and sensitivity to targeted biomolecules; however, these simplified systems fail to incorporate the complex environment of both the diseased tissue and surrounding undiseased tissue. Utilization of endoscopic imaging techniques provides a direct pathway for nondestructive and minimally invasive optical imaging of tissue in vivo. Our group has developed and described in detail a minimally invasive microendoscopic dual-modality imaging system combining OCT and laser induced fluorescence spectroscopy (OCT/LIF) to be used in conjunction with the AOM mouse model of colorectal cancer. ${ }^{50-54}$ This technology allows for simultaneous longitudinal tracking of morphological (OCT) and biochemical (fluorescence) changes as colorectal cancer develops and, in combination with the AOM mouse model, provides a rare opportunity to study cancer development both in vivo and in situ. Also, this technology allows for the superficial imaging of tissue through minimally invasive access. Because of this, lavage, or surface labeling, of this tissue using the contrast agent can be performed, avoiding intravenous injections of high concentrations of potentially toxic contrast agents and providing maximal labeling of the available receptors on the mucosal cell surface. The relatively large size of the contrast agent and topical application also limit the ability of the contrast agent to move into the tissue, further reducing exposure. While studies suggest that accumulated quantum dots do not have cytotoxic effects in vivo, ${ }^{55-57}$ it is still important to minimize this exposure for future use in both animal studies and human clinical imaging.

In this study, QD655-VEGFR2 was used in a longitudinal in vivo study on the ability to visualize VEGFR2 expression using OCT/LIF dual-modality imaging. The goal of this study was to determine if QD655-VEGFR2 could be used in conjunction with OCT/LIF dual-modality imaging to visualize VEGFR2 expression in colorectal tissue. We have previously shown that QD655-VEGFR2 can be used to label adenoma expressing VEGFR2 through in vivo labeling and ex vivo fluorescence imaging with high sensitivity and specificity, and also using the OCT/LIF microendoscope ex vivo. ${ }^{49}$ Quantum dot fluorescence in the colon should coordinate with VEGFR2 expressing adenoma. OCT provided the gold standard for the presence of adenoma with immunohistochemistry (IHC) as a gold standard for VEGFR2 expression. Proper targeting of the contrast agent should yield a stronger fluorescence signal from the diseased regions of the colon than from the surrounding undiseased regions. QD655-VEGFR2 was able to target VGEFR2 expressing diseased areas of colon; however, challenges in fully flushing the unbound contrast agent from the colon before imaging arise when moving from ex vivo imaging to in vivo imaging.

\section{Methods}

\subsection{Contrast Agent Preparation}

Conjugation of QD655 with Streptavidin ${ }^{\circledR}$ (Invitrogen, Grand Island, New York) to anti-VEGFR2 primary antibodies was performed through streptavidin/biotin linking, as previously described. ${ }^{49}$ Briefly, biotinylation of rabbit immunoglobulin $\mathrm{G}(\mathrm{IgG})$ isotype control antibodies (Santa Cruz Biotech, San Diego, California) and anti-VEGFR2 primary antibodies (Abcam, Cambridge, Massachusetts) was performed using the DSB-X Biotin Protein Labeling Kit (Invitrogen). The biotinylated antibodies and the QD655 with Streptavidin ${ }^{\circledR}$ were mixed at a $2: 1$ ratio and incubated for $1.5 \mathrm{~h}$ at room temperature. The resulting contrast agents are called QD655-VEGFR2 (QD655/anti-VEGFR2) and QD655-IC (QD655/isotype control).

\subsection{Mouse Model and Imaging Preparation}

The mouse model used in this study relies on sporadic colorectal carcinogenesis caused by AOM in A/J mice. Twenty-four A/J mice were used in this study, divided into two treatment groups. The experimental group had 13 mice treated with AOM (SigmaAldrich Chemicals, St. Louis, Missouri) (AOM group) and the control group had 11 mice treated with saline (control group). In accordance to a protocol approved by the University of Arizona Institutional Animal Care and Use Committee, AOM $(10 \mathrm{mg} / \mathrm{kg})$ dissolved in saline or saline $(0.2 \mathrm{ml})$ was administered subcutaneously once a week for five weeks, beginning when the mice were six weeks of age. The mice were imaged $3,6,9,12$, and 15 weeks following the final injections for a total of five imaging time points. Mice were placed on a liquid diet (Pedialyte, Abbott Laboratories, Abbott Park, Illinois) for $20 \mathrm{~h}$ before imaging and were anesthetized using ketamine $(0.33 \mathrm{mg} / \mathrm{ml}, \quad 100 \mathrm{mg} / \mathrm{kg})$ and xylazine $(0.033 \mathrm{mg} / \mathrm{ml}$, $10 \mathrm{mg} / \mathrm{kg}$ ) intra peritoneal prior to contrast agent application. Once the mice were fully anesthetized, the colon was gently flushed using warm saline until apparently clear of feces and blood. The colon was prepared for contrast agent application by removing the mucus layer covering the mucosa using the mucolytic agent $\mathrm{N}$-acetylcystine $(1 \%)$. This agent is applied via lavage by filling the colon with the agent and allowing it to incubate for $2 \mathrm{~min}$ before flushing the colon with warm saline. After flushing, the contrast agent was applied via lavage and allowed to incubate for $1 \mathrm{~h}$. The mice were labeled with QD655-VEGFR2 $(15 \mu \mathrm{g} / \mathrm{ml}$ ) (five AOM mice and four control mice), QD655-IC (15 $\mu \mathrm{g} / \mathrm{ml})$ (five AOM mice and three control mice), or saline (three AOM mice and three control mice). After the incubation time, the colon was rinsed with warm saline.

\subsection{Optical Coherence Tomography/Laser-Induced Fluorescence Imaging}

The endoscopic OCT/LIF system has previously been described in detail. ${ }^{53}$ Briefly, longitudinal simultaneous OCT and LIF images were collected at eight evenly spaced circumferential positions rotations (45 deg apart) around the colon, along the distal $30 \mathrm{~mm}$ of the distal colon. A superluminescent diode with a $1300-n m$ center wavelength and 70-nm bandwidth was used as the illumination source for the OCT subsystem (Superlum, Carrigtwohill, Co. Cork, Ireland). This subsystem obtained depth-resolved images $30 \mathrm{~mm}$ long and $2 \mathrm{~mm}$ deep into the tissue with an axial resolution of $8 \mu \mathrm{m}$ and a lateral 
resolution of $18 \mu \mathrm{m}$. An He:Cd laser at $442 \mathrm{~nm}$ was used for the excitation source of QD655 for the LIF subsystem. The resulting emission was captured by a CCD-coupled spectrometer, which measured fluorescence emission intensities between 600 and $700 \mathrm{~nm}$ along the length of the colon. We collected 148 distinct spectra along $30 \mathrm{~mm}$ of colon (spectrum obtained every $200 \mu \mathrm{m}$ ) with eight circumferential rotations [Fig. 2(d)].

\subsection{Image/Data Processing}

OCT images were used to create a binary map of the presence of adenoma in a two-dimensional (2-D) format with the length of the colon on the $x$ axis and the rotation on the $y$ axis. Adenomas were identified according to previously published criteria. ${ }^{58}$ Briefly, each longitudinal OCT image slice was segmented into 36 segments along the $30 \mathrm{~mm}$ length. Each segment was identified as either diseased (adenoma) or undiseased based on the previously published criteria (thickened regional mucosa and/or moderate to marked protrusion of mucosa to more than twice the average regional thickness, moderate to marked signal attenuation and faint or obscured tissue boundaries). Results from all eight longitudinal image slices from a colon were stacked together to create an $8 \times 36$ matrix tumor map (Fig. 1). This procedure was performed for all mice and time points.

Fluorescence emission intensities collected by the LIF subsystem contained spectral information originating from the tissue autofluorescence and the autofluorescence of the system components, as well as the desired signal from the quantum dots [Fig. 2(a)]. Analysis of the spectra identified five separate spectral components, one belonging to QD655 and the others

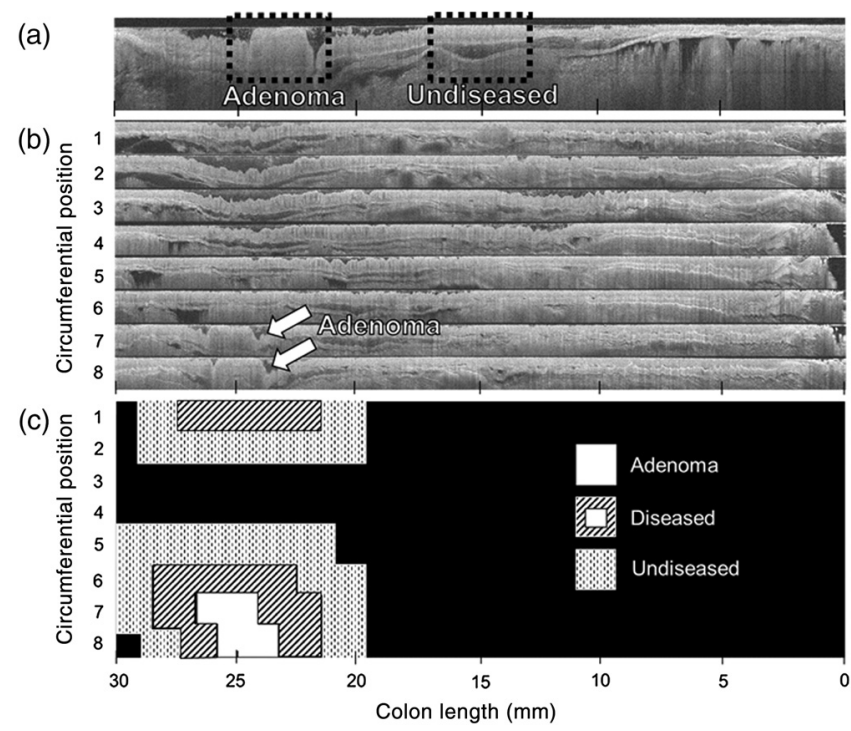

Fig. 1 (a) Optical coherence tomography (OCT) image at one circumferential position. OCT images were graded based on a previously published method for adenoma detection. This method grades segments of the colon as either adenoma or undiseased. (b) All eight radial positions for one mouse and (c) the binary grading of the OCT images from the same mouse to be used in mask formation and analysis. The white area shows how an adenoma transfers from the original OCT images to the tumor map. Then, the adenoma is dilated in all directions and the dilation as well as the original adenoma were considered the diseased area for mask formation. Then the diseased area was dilated again in all directions, and this dilation minus the diseased area is considered the undiseased area for mask formation. originating endogenously from the tissue or system [Fig. 2(b)]. It should be noted that in time point 5 of the experiment, a spectrum with a central wavelength of $605 \mathrm{~nm}$ was detected instead of the expected $655 \mathrm{~nm}$ from QD655. The manufacturer later sent a letter to inform of a mislabeled sample of QD605 being sent to us instead of QD655. For this reason, the spectrum for QD605 was isolated for time point 5. Utilizing a nonlinear least squares curve fit, the quantum dot signal was separated from the collected spectrum for all collected spectra. A 2-D map of the quantum dot fluorescence intensity was formed with the length of the colon on the $x$ axis and the rotation on the $y$ axis, similar to the tumor maps from OCT [Fig. 2(c)]. Unless stated otherwise, these fluorescence intensity maps were downsampled to 36 elements using bicubic interpolation to match the dimensions of the OCT tumor maps for each of the analytical methods described below. The distal $2 \mathrm{~mm}$ were removed from the OCT and LIF maps, as the quantum dots tended to aggregate in the fur and squamous epithelium around the anus. This processing was performed for all mice and time points.

\subsection{Immunohistochemistry}

IHC was performed as previously described. ${ }^{49}$ Briefly, after imaging the colon at the final time point of the study, the colon was explanted, fixed in $2 \%$ formalin, and embedded in paraffin wax. Colon cross-sections were cut at $6 \mu \mathrm{m}$ thicknesses every $500 \mu \mathrm{m}$. The sections were deparafinized and rehydrated and antigen retrieval was performed. Using the same anti-VEGFR2 primary antibody as used to target the quantum dots, but with a goat antirabbit secondary biotinylated for 3,3-diaminobenzidine (DAB) conjugation through streptavidin, VEGFR2 expression in the colons was visualized. All washes were performed using $1 \mathrm{X}$ phosphate buffered saline. The tissues were blocked against nonspecific binding using goat serum and then labeled with the primary anti-VEGFR2 antibody $(2 \mu \mathrm{g} / \mathrm{ml})$ over night at $4^{\circ} \mathrm{C}$. The sections were then blocked for endogenous peroxidase with $3 \%$ hydrogen peroxide before labeling with the secondary goat anti-rabbit IgG biotinylated antibody (1:500) (Vector Labs, Burlingame, California) for $1 \mathrm{~h}$ at room temperature. Last, the sections were incubated with streptavidinHorseradish Peroxidase (Dako, Carpentaria, California), then DAB (brown chromophore) (Dako), and then counterstained using $1 \%$ methyl green.

\subsection{Image/Data Analysis}

As the OCT and LIF images were collected simultaneously, and OCT has previously been proven to have outstanding sensitivity of $95 \%$ to adenoma and a low miss rate of 2 out of 38 adenoma, ${ }^{58}$ the OCT tumor map, along with IHC at the last time point can be used as the gold standard for the presence of adenoma to evaluate the performance of the contrast agent. First, the QD655-VEGFR2 fluorescence emission as measured by LIF was evaluated for its ability to target adenoma. This analysis was performed by comparing the fluorescence intensity of diseased regions and their surrounding undiseased tissue, as well as by determining the sensitivity and specificity at various fluorescence true positive thresholds, according to the following procedure.

Fluorescence intensity comparisons were used to determine if adenoma detected using OCT showed an increased fluorescence signal over surrounding undiseased tissue. The OCT tumor map for each mouse and time point was used to create 


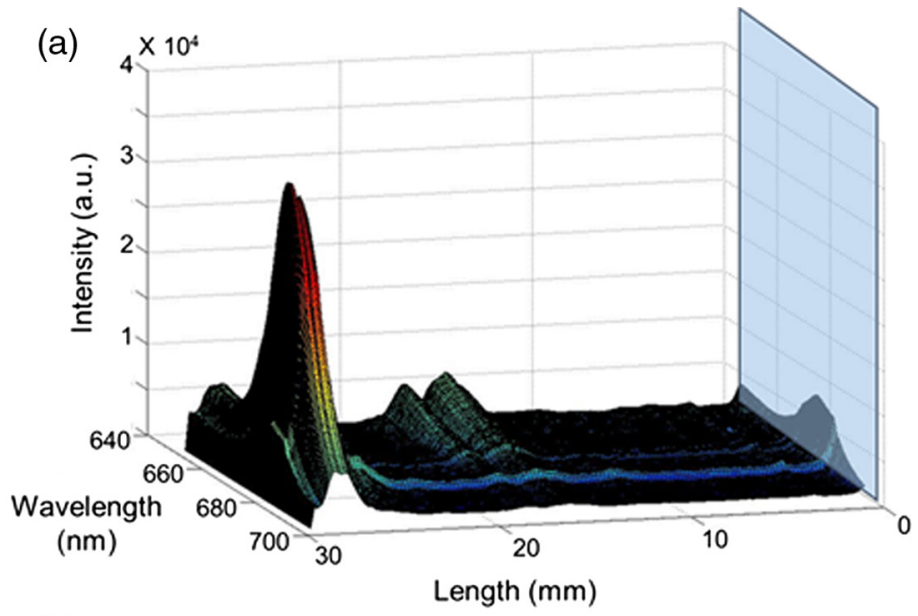

(c)

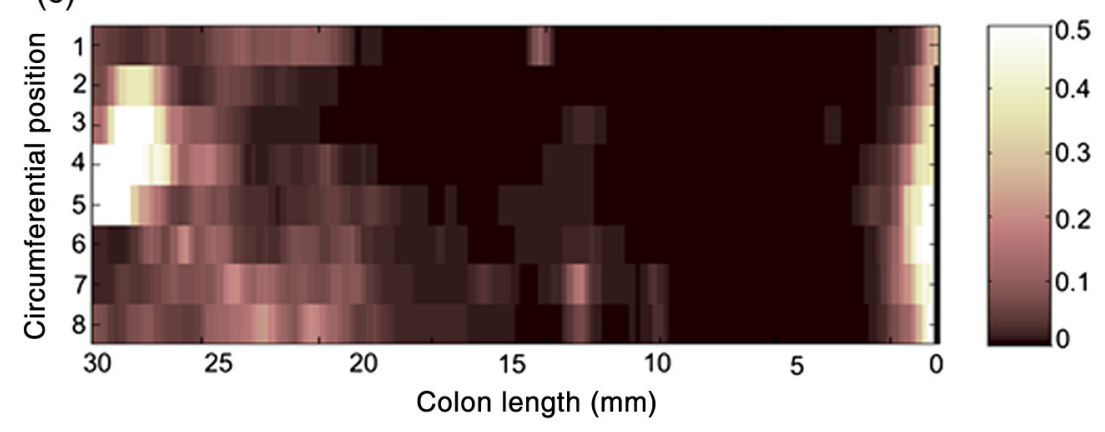

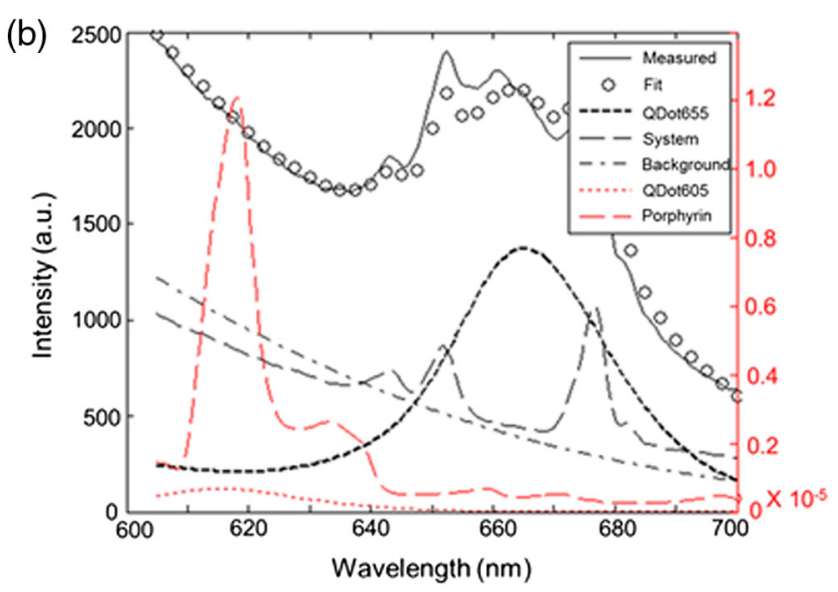

(d)

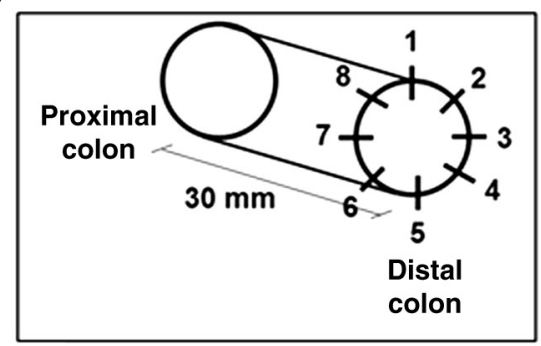

Fig. 2 (a) A surface map of the full fluorescence spectrum from one circumferential position along the length of a colon before the signals are separated. (b) One data point along the length of the colon demonstrating the five distinct spectra that were identified and separated out of the measured spectrum. (c) All eight circumferential positions of the QDot655 (QD655) signal after spectral separation from the measured signal stacked allowing for visualization of the colon as if it were cut along the length between circumferential positions 1 and 8 and splayed open. (d) The position of the eight circumferential image acquisition locations.

a mask for measuring signal intensity in the corresponding quantum dot fluorescence intensity maps [as shown in Fig. 1(c)]. These masks were created by dilating, or increasing the size of, the identified diseased tissue (adenoma) by $2 \mathrm{~mm}$ in both longitudinal directions and 1 pixel (equivalent to $\sim 2 \mathrm{~mm}$ ) in both rotational directions. This dilation was used to accommodate positive labeling that is often found surrounding adenoma boundaries, as shown in our previous study. ${ }^{49}$ The region inside the dilated mask was identified as diseased tissue. The average signal intensity inside was calculated in each individual diseased area in the quantumdot fluorescence intensity maps. Because the tissue around this area was histologically proven to be undiseased, the mask was dilated further by $2 \mathrm{~mm}$ in both longitudinal directions and 1 pixel $(\sim 2 \mathrm{~mm})$ in both rotational directions creating a roughly ring-shaped mask, and the average signal intensity within the mask of the corresponding quantum dot fluorescence intensity maps was computed. The fluorescence signal intensity for each diseased and undiseased area was measured for all mice and time points. The average fluorescence intensity for each contrast agent/treatment group combination was then determined by averaging all of the diseased areas together and all of the undiseased areas together for that group. Statistical significance between diseased and undiseased areas and between contrast agent/treatment group combinations was determined using a one-tailed Student's $t$ test.

To compare the average fluorescence intensities measured in this in vivo study to the average fluorescence intensity values measured in our previously published ex vivo study, ${ }^{49}$ both the current and previous fluorescence intensity values were normalized to the highest value, which in both cases was from the diseased regions of the QD655-VEGFR2 labeled, AOM-treated colons.

To determine the classification performance (sensitivity and specificity), a variable threshold was applied to the fluorescence intensity maps. This threshold was identical for each LIF image and ranged from an intensity level of 0 to a maximum of $>95 \%$ of intensity values. A true positive (TP) was defined as $>20 \%$ of the pixels within a diseased region being above the threshold, with a false negative (FN) occurring if this value was $<20 \%$. A true negative (TN) was determined when $<20 \%$ of the pixels within the undiseased region were above threshold, with a false positive (FP) occurring if the value was $>20 \%$. Each region was classified as TP, TN, FP, or FN. Specificity was calculated using the measured values from the control mice and sensitivity was calculated using the AOM mice, for each time point and contrast agent group.

As additional analysis, the quantum dot fluorescence intensity spatial distribution was evaluated to determine if the quantum dots were accumulating in any particular region of the colon of AOM-treated mice. A one-dimensional vector of intensity values along the length of the colon for each mouse was formed by integrating data in the circumferential dimension. A corresponding integration was performed on the binary OCT tumor maps to determine the distribution of adenoma along 
the length of the colon. The resulting profiles show the fluorescence distribution for each time point and each contrast agent group, or for individual mice in each time point. The colon was then divided into thirds corresponding to the proximal, middle, and distal regions, and the percent of the total fluorescence signal in each region was calculated. This procedure was repeated to evaluate the distribution in the circumferential dimension.

\section{Results/Discussion}

Table 1 includes a count of all mice that started and completed the study, the adenoma count at the final time point, and the number of diseased/undiseased region pairs used in the analysis (which includes the sum of all region pairs at all time points, including in some cases mice that did not survive to the final time point). Three mice died as a result of complications from anesthesia. The other five mice were euthanized due to excessive tumor burden/bleeding and intestinal impactions. No salinetreated (control) mice developed adenoma during the study. Adenoma appeared in four AOM-treated mice by time point 3 , in six AOM-treated mice by time point 4 , and in all but one AOM-treated mouse by time point 5 . In the AOM-treated group, the QD655-VEGFR2 labeled group had 14 measured adenomas by the final time point, the QD655-IC labeled group had 9 measured adenomas by the final time point, and the saline labeled group had 7 measured adenomas by the final time point.

Using the tumor maps generated through OCT, the average fluorescence intensity from the diseased tissue and the surrounding undiseased tissue was measured for each contrast agent/ treatment group combination. As seen in Fig. 3, the fluorescence intensity for the diseased regions of QD655-VEGFR2 labeled, AOM-treated colons was 3.2 times stronger than that of the

Table 1 This table includes the total number of mice, the number of mice surviving at time point 5 , the total number of adenoma that developed by time point 5 , and the total number of diseased and undiseased regions measured in each contrast agent/treatment group combination. The number of diseased and undiseased regions measured includes each adenoma at each time point, meaning an adenoma at time point 4 and the same adenoma at time point 5 were considered as two separate adenomas. Also, if the diseased regions of two adenomas in one colon overlapped after dilation, they were considered one diseased area. There was one undiseased region measured for every diseased region measured.

\begin{tabular}{|c|c|c|c|c|c|}
\hline $\begin{array}{l}\text { Contrast } \\
\text { agent }\end{array}$ & Treatment & $\begin{array}{l}\text { Starting } \\
\text { number of } \\
\text { mice }\end{array}$ & $\begin{array}{l}\text { Surviving } \\
\text { mice at } \\
\text { TP5 }\end{array}$ & $\begin{array}{l}\text { Number } \\
\text { of } \\
\text { adenoma } \\
\text { at TP5 }\end{array}$ & $\begin{array}{c}\text { Number of } \\
\text { diseased/ } \\
\text { undiseased } \\
\text { regions } \\
\text { measured }\end{array}$ \\
\hline \multirow{2}{*}{$\begin{array}{l}\text { QD655- } \\
\text { VEGFR2 }\end{array}$} & $\mathrm{AOM}$ & 5 & 4 & 9 & 14 \\
\hline & Saline & 4 & 3 & 0 & 0 \\
\hline \multirow{2}{*}{$\begin{array}{l}\text { QD655- } \\
\text { IC }\end{array}$} & $\mathrm{AOM}$ & 5 & 3 & 8 & 9 \\
\hline & Saline & 4 & 2 & 0 & 0 \\
\hline \multirow[t]{2}{*}{ Control } & $\mathrm{AOM}$ & 3 & 2 & 3 & 7 \\
\hline & Saline & 3 & 3 & 0 & 0 \\
\hline
\end{tabular}

QD655, QDot655; VEGFR2, vascular endothelial growth factor receptor 2; IC, isotype control; AOM, azoxymethane. surrounding undiseased tissue suggesting that the contrast agent is correctly labeling the diseased areas. Intensities were not significantly different, however, due to a high standard deviation in both data sets $(p=0.12)$. The average fluorescence of the diseased regions of QD655-VEGFR2 labeled colons was 4.8 times stronger than the diseased regions of QD655-IC labeled colons, suggesting that the binding was specific. Again, this difference was not significant due to large standard deviations $(p=0.09)$. There was no significant difference in the average intensity of diseased and undiseased regions of QD655IC labeled colons, suggesting that this nonspecific agent was passively accumulating in the colon. The average intensities for all other contrast agent/treatment group combinations were low. The minimal fluorescence intensity registered from both AOM-treated and control colons lavaged with only saline represents the low level of tissue autofluorescence. Low levels of fluorescence from control colons lavaged with QD655VEGFR2 and QD655-IC likely represent passive accumulation of the contrast agent in the colon. A significant difference in average fluorescence intensity was seen between diseased areas of QD655-VEGFR2 labeled, AOM-treated colons and diseased areas of saline labeled, AOM-treated colons (factor of $48, p=0.049$ ). Also, a significant difference was seen between undiseased regions of QD655-VEGFR2 labeled AOM-treated colons and QD655-VEGFR2 labeled control colons (factor of 5.6, $p=0.032$ ), indicating that there is significantly more contrast agent accumulating in the undiseased tissue of AOM-treated colon than control colons.

Comparison of data from this in vivo study and our previously published ex vivo study ${ }^{49}$ shows strikingly similar trends, as seen in Fig. 4, in which the average fluorescence intensities from both the in vivo study and the ex vivo study are plotted together. The ex vivo study results demonstrated statistically significant differences between groups, which in this study failed to achieve significance due to large standard deviations. The difference between our current study and the previous study lies in the in vivo imaging and data processing. In the previous ex vivo study, colons were excised and splayed open for imaging, allowing thorough rinsing of the tissue and greatly reducing the unbound contrast agent present in the colon before imaging was conducted. Although most imaging was performed utilizing a fluorescence microscope, an example ex vivo imaging using the OCT/LIF microendoscope employed in this study showed strong QD655-VEGFR2 signal in the tumor, but nearly an undetectable signal in undiseased tissue, suggesting that differences in the significance in the data are likely attributed to the differences in the ability to fully rinse the unbound contrast agents from the colons when imaging in vivo, rather than being attributable to the endoscope. In vivo rinsing of the mouse colon poses challenges in removing unbound contrast agent from the mucosa due to the small diameter of the colon and the tendency of fluid to accumulate in the more proximal colon, particularly as adenoma develops and the lumen of the colon becomes blocked.

As seen in Fig. 4, the difference in the average fluorescence intensity between the diseased and undiseased regions of QD655-VEGFR2 labeled, AOM-treated colons was similar, as was the difference in the average intensity of the QD655VEGFR2 labeled, AOM-treated diseased regions and the QD655-IC labeled, AOM-treated diseased regions for the in vivo and ex vivo studies. In the in vivo study, the average fluorescence intensity of the undiseased areas of the colons in the 


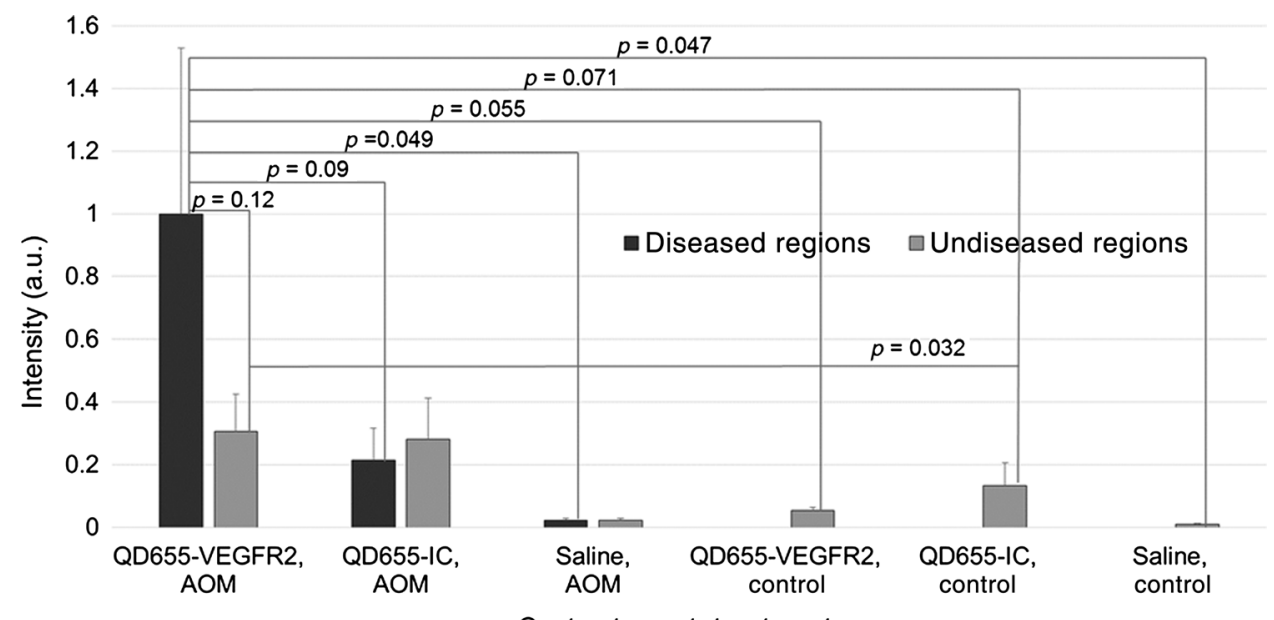

Fig. 3 Fluorescence intensity measurements of each diseased region and the surrounding undiseased regions for each colon were taken. All diseased regions or all undiseased regions for all colons of mice labeled with a particular contrast agent and in a particular treatment group were averaged together for a representative signal intensity. All tumors from all time points were combined for each group. Standard error is displayed, as well as $p$ values from the Student's $t$ test. $N$ values can be taken from Table 1 as the number of diseased/undiseased regions measured.

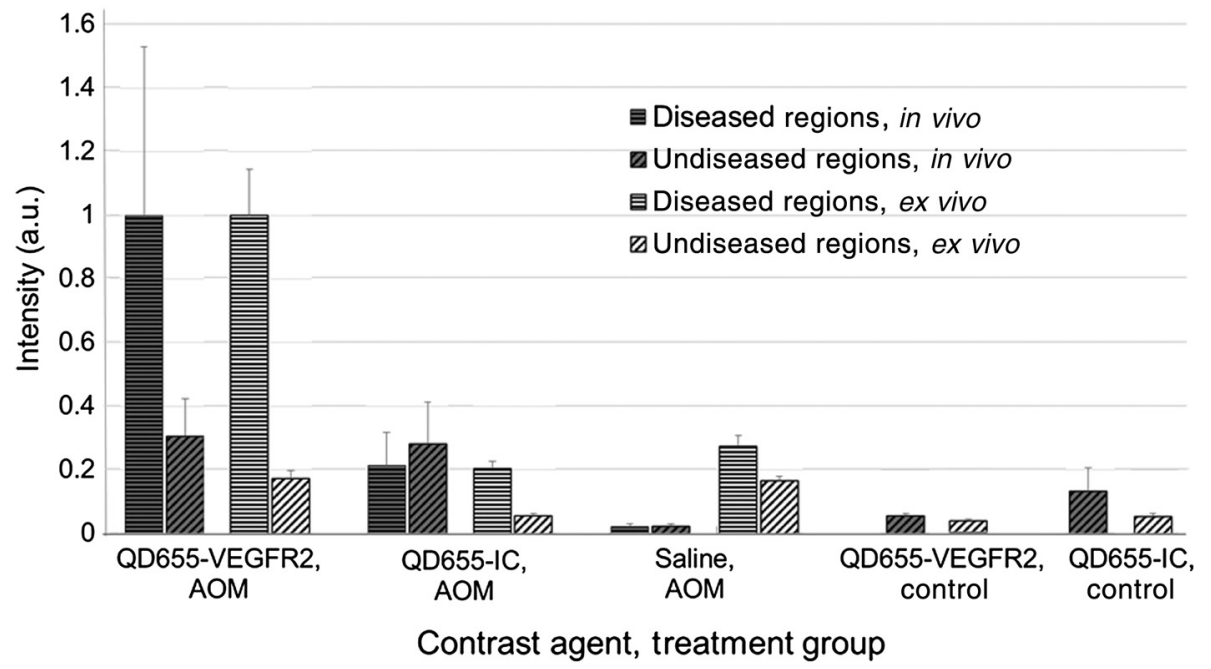

Fig. 4 The fluorescence intensity results from the current in vivo study compared to those from the previous ex vivo study. Both the diseased and undiseased tissues for each contrast agent/treatment group are represented. Error bars represent standard error.

QD655-VEGFR2 and QD655-IC labeled, AOM-treated colons and control colons was higher than these regions in the ex vivo study, suggesting more passive accumulation. It is interesting to note that the ex vivo study captured more autofluorescence from the tissue (see in saline labeled tissue), since the ex vivo study did not utilize spectral separation of the quantum dot and autofluorescence signals.

Classification accuracy in the current study data for QD655VEGFR2 labeled colons revealed a low diagnostic performance, as might be expected given the inability to achieve statistical significance between diseased and undiseased regions. Maximum classification performance (sensitivity + specificity) was $140 \%$ (perfect performance would be at $200 \%$ ). A typical sensitivity was $40 \%$ with 80 to $85 \%$ specificity. In the previous ex vivo study, a sensitivity of $85.7 \%$ at a specificity of $91.3 \%$ was obtained. Given the similarities in average trends between in vivo and ex vivo studies, but the large standard deviations in the in vivo study, it was speculated that challenges in fully flushing the unbound contrast agent from the colons in vivo account for the differences in classification accuracy.

The distribution of the fluorescence signal along the length of the colon and at each circumferential image location for each time point and contrast agent supports this supposition. The fluorescence intensity is typically higher in the proximal end of the colon, as well as around the anus (Fig. 5, near 30 and $0 \mathrm{~mm}$ ). The nonspecific fluorescence at the anus was mostly removed by excluding the distal $2 \mathrm{~mm}$, which was contaminated with fluorescence from dyed fur and squamous epithelium. Data from QD655-IC labeled colons had a similar trend in fluorescence intensity distribution without correlation to tumor locations. Circumferential distributions revealed that in several time points, there is an accumulation of fluorescence signal at 

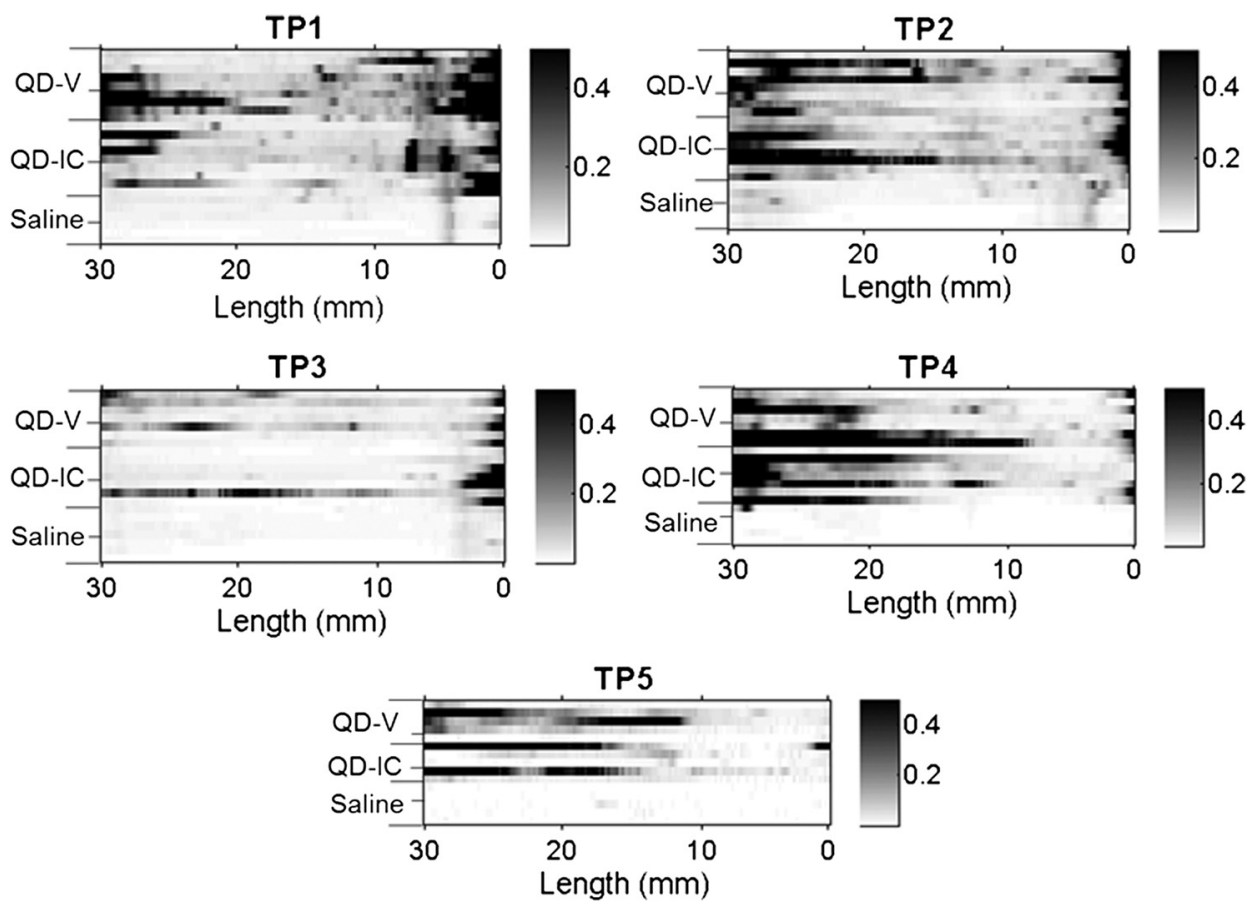

Fig. 5 Fluorescence intensity at each longitudinal position was summed circumferentially for each mouse to demonstrate the fluorescence distribution along the length of the colon. This was performed for all living mice at each time point. Each treatment group [QD-V = QD655-vascular endothelial growth factor receptor 2 (VEGFR2), QD-IC = QD655-isotype control] is indicated on the $y$ axis, with the small line in the middle of each group indicating where the azoxymethane (AOM) treated mice stop and the control mice start. A value of 0.5 is set as the maximum display value, causing all values $>0.5$ to be saturated.

a ventral position (in the direction of gravity, data not shown). The inability to completely remove unbound contrast agent from the colon is most likely the key contributor to this nonspecific fluorescence signal distribution along the length of the colon and in the direction of gravity, causing a large signal variation.

While evidence suggests that contrast agent accumulation occurs due to the difficulty in flushing the small mouse colon, it is important to also note that some of the accumulation of signal in the nondiseased regions may be attributed to actual changes in VEGFR2 expression throughout AOM-treated colons. AOM-treated undiseased areas are not normal compared to saline-treated control colons. They have been shown to have abnormal crypt structure ${ }^{59}$ and have an increased expression of VEGFR2 at a level between that of adenoma and control tissue (Fig. 6). There are also some changes in the morphology of the undiseased tissue in AOM-treated colon and control colons (e.g., thicker mucosa), which may contribute to the increased accumulation of contrast agent in the undiseased regions. Also, it is for this reason that the control colons (no AOM injections) were used to calculate the specificity of the contrast agent, demonstrating the true performance in completely normal tissue. Because the average relative fluorescence signal from the QD655-VEGFR2 labeled undiseased regions in AOM-treated mice was about two times lower in the ex vivo study than the (a)

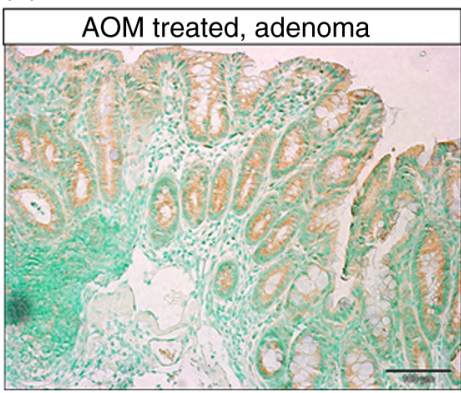

(b)

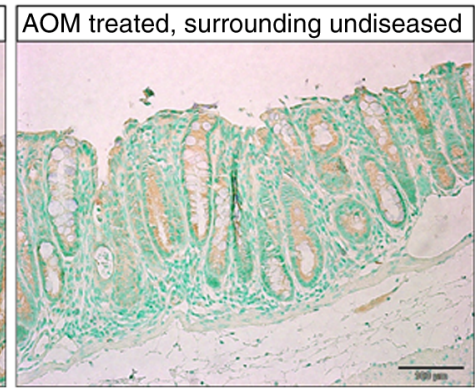

(c)

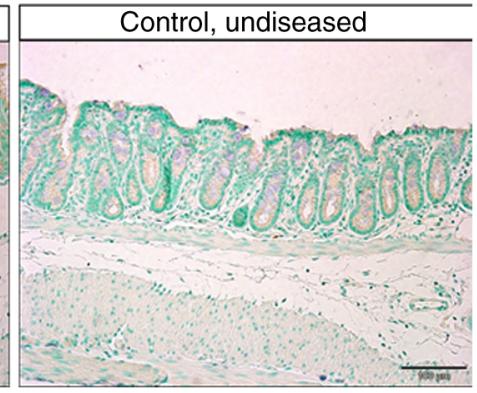

Fig. 6 Immunohistochemistry for VEGFR2 was performed on all colons after time point 5 imaging. Adenomas from AOM mice (a) are different in both VEGFR2 expression and morphology than undiseased tissue from control mice (c). This difference is less notable for the undiseased tissue in the same AOM treated mice (b), potentially contributing to nonspecific accumulation of contrast agent in undiseased tissue. Scale bar: $100 \mu \mathrm{m}$. 
in vivo study, the elevation in VEGFR2 expression in AOMtreated colons was likely not the major contributor to the fluorescence signal found in undiseased tissue in this study.

This study provides further evidence that QD655-VEGFR2 contrast agent can selectively target VEGFR2 expressing adenoma and that the OCT/LIF imaging system is capable of detecting this signal due to the narrow emission spectrum of the quantum dots allowing the ease of separation from competing signals. In future studies, more thorough flushing of the unbound contrast agent is expected to result in a reduction in nonspecific signal as well as standard deviation of measured fluorescence intensity values, leading to increased significance between contrast agent/treatment groups and improved classification of diseased and undiseased regions. Use of a miniature balloon catheter that blocked the proximal colon and the constrained contrast agent and flushing solutions to the distal colon only could greatly aid complete removal of unbound contrast agent from the colon.

\section{Conclusions}

Through this study, and the previously published ex vivo study, the ability of the QD655-VEGFR2 contrast agent to target VEGFR2 expressing adenoma in an AOM-treated mouse colon has been demonstrated. In this in vivo imaging study, the average intensity of the diseased regions of colons labeled with QD655-VEGFR2 was 3.2 times higher than the intensity from surrounding undiseased tissue in the same colons and 4.8 times higher than the intensity from the diseased regions of colons labeled with QD655-IC. This is similar to the results obtained in the ex vivo study. The main challenge of moving from ex vivo imaging to in vivo imaging in this application is the nonspecific accumulation of the contrast agent in the colon, providing a variable background that increased standard deviations and precluded statistical significance in the fluorescence intensity data. It is notable that while the small diameter of the mouse colon provides a great challenge in flushing of the colon, the larger diameter of the human colon would mitigate this concern. While improvements in flushing of the unbound contrast agent will need to be investigated, this study demonstrates the ability of the OCT/LIF system to detect the contrast agent in vivo, providing a minimally invasive in vivo imaging technique for visualizing molecular expression of colon adenoma.

\section{Acknowledgments}

Research reported in this publication was supported by the National Cancer Institute and the National Institute of Heart, Lung and Blood of the National Institutes of Health under award numbers R01CA109835 and T32HL007955. The content is solely the responsibility of the authors and does not necessarily represent the official views of the National Institutes of Health. We would also like to acknowledge funding from the NSF GTEAMS STEM in K-12 Graduate Fellowship \#081234. Last, we acknowledge Faith Rice and Dr. Sarah Leung for their expertise and assistance.

\section{References}

1. M. F. Penet et al., "Molecular imaging of the tumor microenvironment for precision medicine and theranostics," Adv. Cancer Res. 124, 235256 (2014).
2. B. E. Schaafsma et al., "The clinical use of indocyanine green as a nearinfrared fluorescent contrast agent for image-guided oncologic surgery," J. Surg. Oncol. 104, 323-332 (2011).

3. S. L. Troyan et al., "The FLARE intraoperative near-infrared fluorescence imaging system: a first-in-human clinical trial in breast cancer sentinel lymph node mapping," Ann. Surg. Oncol. 16, 2943-2952 (2009).

4. R. Bhuvaneswari et al., "Evaluation of hypericin-mediated photodynamic therapy in combination with angiogenesis inhibitor bevacizumab using in vivo fluorescence confocal endomicroscopy," J. Biomed. Opt. 15, 011114 (2010).

5. A. A. Neves and K. M. Brindle, "Assessing responses to cancer therapy using molecular imaging," Biochim. Biophys. Acta 1766, 242-261 (2006).

6. T. Hussain and Q. T. Nguyen, "Molecular imaging for cancer diagnosis and surgery," Adv. Drug Deliv. Rev. 66, 90-100 (2014).

7. N. Khemthongcharoen, "Advances in imaging probes and optical microendoscopic imaging techniques for early in vivo cancer assessment," Adv. Drug Deliv. Rev. 74, 53-74 (2014).

8. American Cancer Society, Cancer Facts \& Figures 2015, American Cancer Society, Atlanta (2015).

9. H. Cho et al., "In vivo cancer imaging by poly(ethylene glycol)-b-poly (epsilon-caprolactone) micelles containing a near-infrared probe," Nanomedicine 8, 228-236 (2012).

10. M. Goetz et al., "In vivo molecular imaging with cetuximab, an antiEGFR antibody, for prediction of response in xenograft models of human colorectal cancer," Endoscopy 45, 469-477 (2013).

11. L. Liu et al., "In vivo molecular imaging of gastric cancer in humanmurine xenograft models with confocal laser endomicroscopy using a tumor vascular homing peptide," Cancer Lett. 356, 891898 (2015).

12. B. Paudyal et al., "Positron emission tomography imaging and biodistribution of vascular endothelial growth factor with $64 \mathrm{Cu}-1 \mathrm{labeled}$ bevacizumab in colorectal cancer xenografts," Cancer Sci. 102, 117-121 (2011).

13. A. Pichorner et al., "In vivo imaging of colorectal cancer growth and metastasis by targeting MACC1 with shRNA in xenografted mice," Clin. Exp. Metastasis 29, 573-583 (2012).

14. K. K. Frese and D. A. Tuveson, "Maximizing mouse cancer models," Nat. Rev. Cancer 7, 654-658 (2007).

15. A. Kamb, "What's wrong with our cancer models?" Nat. Rev. Drug Discov. 4, 161-165 (2005).

16. C. Neufert, C. Becker, and M. F. Neurath, "An inducible mouse model of colon carcinogenesis for the analysis of sporadic and inflammationdriven tumor progression," Nat. Protoc. 2, 1998-2004 (2007).

17. V. Biju et al., "Bioconjugated quantum dots for cancer research: present status, prospects and remaining issues (vol 28, pg 199, 2010)," Biotechnol. Adv. 29, 259-260 (2011).

18. M. Fang et al., "Quantum dots for cancer research: current status, remaining issues, and future perspectives," Cancer Biol. Med. 9, 151-163 (2012).

19. X. Gao et al., "In vivo molecular and cellular imaging with quantum dots," Curr. Opin. Biotechnol. 16, 63-72 (2005).

20. E. Cassette et al., "Design of new quantum dot materials for deep tissue infrared imaging," Adv. Drug Deliv. Rev. 65, 719-731 (2013).

21. H. Mattoussi, G. Palui, and H. B. Na, "Luminescent quantum dots as platforms for probing in vitro and in vivo biological processes," $A d v$. Drug Deliv. Rev. 64, 138-166 (2012).

22. J. K. Jaiswal et al., "Long-term multiple color imaging of live cells using quantum dot bioconjugates," Nat. Biotechnol. 21, 47-51 (2003).

23. D. S. Lidke et al., "Reaching out for signals: filopodia sense EGF and respond by directed retrograde transport of activated receptors," J. Cell Biol. 170, 619-626 (2005).

24. K. C. Weng et al., "Targeted tumor cell internalization and imaging of multifunctional quantum dot-conjugated immunoliposomes in vitro and in vivo," Nano Lett. 8, 2851-2857 (2008).

25. X. Wu et al., "Immunofluorescent labeling of cancer marker Her2 and other cellular targets with semiconductor quantum dots," Nat. Biotechnol. 21, 41-46 (2003).

26. M. V. Yezhelyev et al., "In situ molecular profiling of breast cancer biomarkers with multicolor quantum dots," Adv. Mater. 19, 3146-3151 (2007). 
27. K. T. Yong et al., "Imaging pancreatic cancer using bioconjugated InP quantum dots," ACS Nano 3, 502-510 (2009).

28. M. E. Akerman et al., "Nanocrystal targeting in vivo," Proc. Natl. Acad. Sci. USA 99, 12617-12621 (2002).

29. B. Ballou et al., "Sentinel lymph node imaging using quantum dots in mouse tumor models," Bioconjug. Chem. 18, 389-396 (2007).

30. W. Cai et al., "Peptide-labeled near-infrared quantum dots for imaging tumor vasculature in living subjects," Nano Lett. 6, 669-676 (2006).

31. J. Gao et al., "Ultrasmall near-infrared non-cadmium quantum dots for in vivo tumor imaging," Small 6, 256-261 (2010).

32. J. Gao et al., "In vivo tumor-targeted fluorescence imaging using nearinfrared non-cadmium quantum dots," Bioconjug. Chem. 21, 604-609 (2010).

33. X. Gao et al., "In vivo cancer targeting and imaging with semiconductor quantum dots," Nat. Biotechnol. 22, 969-976 (2004).

34. S. Kim et al., "Near-infrared fluorescent type II quantum dots for sentinel lymph node mapping," Nat. Biotechnol. 22, $93-97$ (2004).

35. D. R. Larson et al., "Water-soluble quantum dots for multiphoton fluorescence imaging in vivo," Science 300, 1434-1436 (2003).

36. Y. Park et al., "Spraying quantum dot conjugates in the colon of live animals enabled rapid and multiplex cancer diagnosis using endoscopy," ACS Nano 8, 8896-8910 (2014).

37. M. Stroh et al., "Quantum dots spectrally distinguish multiple species within the tumor milieu in vivo," Nat. Med. 11, 678-682 (2005).

38. H. Tada et al., "In vivo real-time tracking of single quantum dots conjugated with monoclonal anti-HER2 antibody in tumors of mice," Cancer Res. 67, 1138-1144 (2007).

39. K. Yang et al., "In-vivo imaging of oral squamous cell carcinoma by EGFR monoclonal antibody conjugated near-infrared quantum dots in mice," Int. J. Nanomed. 6, 1739-1745 (2011).

40. K. Yang et al., "In vivo and in situ imaging of head and neck squamous cell carcinoma using near-infrared fluorescent quantum dot probes conjugated with epidermal growth factor receptor monoclonal antibodies in mice," Oncol. Rep. 27, 1925-1931 (2012)..

41. K. Fujimoto et al., "Expression of two angiogenic factors, vascular endothelial growth factor and platelet-derived endothelial cell growth factor in human pancreatic cancer, and its relationship to angiogenesis," Eur. J. Cancer 34, 1439-1447 (1998).

42. D. J. Hicklin and L. M. Ellis, "Role of the vascular endothelial growth factor pathway in tumor growth and angiogenesis," J. Clin. Oncol. 23, 1011-1027 (2005).

43. J. Y. Kim et al., "Prognostic significance of epidermal growth factor receptor and vascular endothelial growth factor receptor in colorectal adenocarcinoma," APMIS 119, 449-459 (2011).

44. Y. Takahashi et al., "Significance of vessel count and vascular endothelial growth factor and its receptor (KDR) in intestinal-type gastric cancer," Clin. Cancer Res. 2, 1679-1684 (1996).

45. Y. Takahashi et al., "Expression of vascular endothelial growth-factor and its receptor, Kdr, correlates with vascularity, metastasis, and proliferation of human colon-cancer," Cancer Res. 55, 3964-3968 (1995).

46. L. M. Ellis and D. J. Hicklin, "VEGF-targeted therapy: mechanisms of anti-tumour activity," Nat. Rev. Cancer 8, 579-591 (2008).

47. N. Ferrara, K. J. Hillan, and W. Novotny, "Bevacizumab (Avastin), a humanized anti-VEGF monoclonal antibody for cancer therapy," Biochem. Biophys. Res. Commun. 333, 328-335 (2005).

48. J. C. Lee, "Prognostic value of vascular endothelial growth factor expression in colorectal cancer patients," Eur. J. Cancer 36, 748-753 (2000).

49. J. L. Carbary-Ganz, J. K. Barton, and U. Utzinger, "Quantum dots targeted to vascular endothelial growth factor receptor 2 as a contrast agent for the detection of colorectal cancer," J. Biomed. Opt. 19, 086003 (2014).

50. L. P. Hariri et al., "Simultaneous optical coherence tomography and laser induced fluorescence imaging in rat model of ovarian carcinogenesis," Cancer Biol. Ther. 10, 438-447 (2010).

51. L. P. Hariri et al., "Endoscopic optical coherence tomography and laserinduced fluorescence spectroscopy in a murine colon cancer model," Lasers Surg. Med. 38, 305-313 (2006).

52. S. J. Leung, P. S. Rice, and J. K. Barton, "In vivo molecular mapping of the tumor microenvironment in an azoxymethane-treated mouse model of colon carcinogenesis," Lasers Surg. Med. 47, 40-49 (2015).

53. A. R. Tumlinson et al., "Miniature endoscope for simultaneous optical coherence tomography and laser-induced fluorescence measurement," Appl. Opt. 43, 113-121 (2004).

54. A. M. Winkler et al., "In vivo, dual-modality OCT/LIF imaging using a novel VEGF receptor-targeted NIR fluorescent probe in the AOMtreated mouse model," Mol. Imaging Biol. 13, 1173-1182 (2011).

55. T. S. Hauck et al., "In vivo quantum-dot toxicity assessment," Small $\mathbf{6}$, 138-144 (2010).

56. M. Vibin et al., "Biokinetics and in vivo distribution behaviours of silica-coated cadmium selenide quantum dots," Biol. Trace Elem. Res. 142, 213-222 (2011).

57. L. Ye et al., "A pilot study in non-human primates shows no adverse response to intravenous injection of quantum dots," Nat. Nanotechnol. 7, 453-458 (2012).

58. L. P. Hariri et al., "Serial endoscopy in azoxymethane treated mice using ultra-high resolution optical coherence tomography," Cancer Biol. Ther. 6, 1753-1762 (2007).

59. M. R. Keenan et al., "Dual optical modality endoscopic imaging of cancer development in the mouse colon," Lasers Surg. Med. 47, 30-39 (2015).

Jordan L. Carbary-Ganz is currently a veterinary medicine student at the University of Florida. She holds a PhD and BS in biomedical engineering from the University of Arizona and University of Arkansas, respectively. Her research focuses on novel contrast mechanisms for colon cancer screening.

Weston A. Welge is a doctoral student at the College of Optical Sciences at the University of Arizona. He received his BS in electrical and computer engineering and BA in history from the University of Colorado at Boulder and MS in optical sciences from the University of Arizona. His research focuses on the development of miniature optical endoscopes and novel applications of optical coherence tomography for early cancer detection.

Jennifer K. Barton is currently a professor of biomedical engineering, electrical and computer engineering, and optical sciences at the University of Arizona. She has served as the assistant director of the $\mathrm{BIO5}$ Institute and interim vice president for research. She is a fellow of SPIE and the American Institute for Medical and Biological Engineering. Her work on development of miniature multimodality optical endoscopes and light-tissue interaction has led to over 90 peer-reviewed journal papers.

Urs Utzinger is currently an associate professor and associate department head in Biomedical Engineering at the University of Arizona. He holds appointments in Ob/Gyn, Optical Sciences, Electrical and Computer Engineering and the $\mathrm{BIO5}$ Institute. His work on fiberoptic probes, diagnostic spectroscopy and microcopy has led to over 70 publications in peer-reviewed journals. 